\title{
Anesthetic management in an adult moyamoya disease patient undergoing mitral valve plasty for severe mitral regurgitation
}

\author{
Kazutomo Saito ${ }^{1 *}$, Hiroaki Toyama ${ }^{1}$, Yutaka Ejima ${ }^{2}$ and Masanori Yamauchi ${ }^{3}$
}

\begin{abstract}
Background: Despite several previous reports, there are no established procedures for intraoperative management in moyamoya disease patients requiring cardiac surgery.

Case presentation: Herein, we report the case of a 42-year-old man who was scheduled to undergo mitral valve plasty for severe mitral regurgitation. He had been diagnosed with moyamoya disease on the onset of cerebral ischemia at 41 years of age. During the cardiac surgical procedure, the patient was maintained on inhalation anesthesia with 1 to $1.5 \%$ sevoflurane. Sevoflurane causes cerebral vasodilation followed by increased cerebral blood flow, and moreover we expected a sevoflurane preconditioning-induced neuroprotective effect. In addition, we used pulsatile perfusion support to maintain cerebral circulation with intra-aortic balloon pumping during the cardiopulmonary bypass. We aimed to keep the mean arterial pressure constantly above $70 \mathrm{mmHg}$. We were able to maintain regional cerebral oxygen saturation at $80 \%$ of the baseline value, and could not detect the progression of neurological deficits using follow-up brain single photon emission computed tomography. The patient was discharged 16 days after admission.

Conclusions: The details of the clinical course of his case will add to our knowledge regarding intraoperative management options in moyamoya disease patients requiring cardiac surgery. We suggest that pulsatile blood flow supported by intra-aortic balloon pumping and sevoflurane anesthesia for increasing cerebral blood flow and for possible neuroprotection may be efficacious for anesthetic management of moyamoya disease patients.
\end{abstract}

Keywords: Moyamoya disease, Cardiopulmonary bypass, Intra-aortic balloon pumping, Sevoflurane,

Preconditioning

\section{Background}

Moyamoya disease (MMD) is a chronic cerebrovascular disorder characterized by steno-occlusive changes of the terminal portion of the internal carotid arteries and the development of a network of abnormal collateral vessels [1]. In MMD patients with severe cerebrovascular disorders, prevention of cerebral ischemia or cerebral hemorrhage during cardiac surgery involving cardiopulmonary bypass (CPB) is extremely difficult because autoregulation of cerebral

\footnotetext{
*Correspondence: kazutomo0815@gmail.com

'Department of Anesthesiology, Tohoku University Hospital, 1-1 Seiryomachi,

Aoba-ku, Sendai 980-8574, Japan

Full list of author information is available at the end of the article
}

blood flow is impaired. Several previous reports have described intraoperative management in MMD patients requiring cardiac surgery [2-4], but there is no established evidence regarding anesthetic agents for maintenance of general anesthesia, effects of pulsatile perfusion, appropriate cerebral perfusion pressure and arterial carbon dioxide partial pressure $\left(\mathrm{PaCO}_{2}\right)$ during $\mathrm{CPB}$.

Herein, we report the successful management of a patient who had right hemiplegia due to MMD, and who underwent mitral valve plasty for severe mitral regurgitation (MR) without deterioration of neurological function. 


\section{Case presentation}

A 41-year-old man (height $171.5 \mathrm{~cm}$, body weight $67 \mathrm{~kg}$ ) was transferred to the regional medical center due to right hemiplegia and aphagia. Cerebral magnetic resonance imaging revealed cerebral infarction caused by occlusion of the left middle cerebral artery, while cerebral magnetic resonance angiography showed the development of a network of abnormal collateral vessels. Hence, the patient was diagnosed with MMD.

Before cerebral revascularization surgery, severe MR (III/IV) due to the prolapse of the P2 leaflet in the mitral valve was indicated by transthoracic echocardiography. Cardiac catheterization indicated elevated pulmonary arterial pressure (PAP) (systolic/diastolic/mean: 86/33/ $60 \mathrm{mmHg}$ ) and pulmonary capillary wedge pressure (32 $\mathrm{mmHg}$ ) at the systemic arterial pressure of 120/83/ $102 \mathrm{mmHg}$. Hence, the patient was admitted to our university center for the surgical treatment of MR.

Preoperative single-photon emission computed tomography revealed reduced cerebral blood flow in the left cerebral hemisphere (especially in the external left frontal cortex; Fig. 1). Neurosurgeons at our center judged that the patient did not have an indication for cerebral revascularization surgery, which is used to prevent ischemic complications during the perioperative period of mitral valve plasty. This was because his left frontal lobe showed extensive cerebral infarction and no cerebral infarction symptoms were observed in the right cerebral hemisphere. Therefore, mitral valve plasty without cerebral revascularization was chosen.

In the operation room, the patient's monitoring of electrocardiogram, oxygen saturation, systemic arterial pressure via right radial artery catheter, bispectral index, and regional cerebral oxygen saturation $\left(\mathrm{rSO}_{2}\right)$ at the

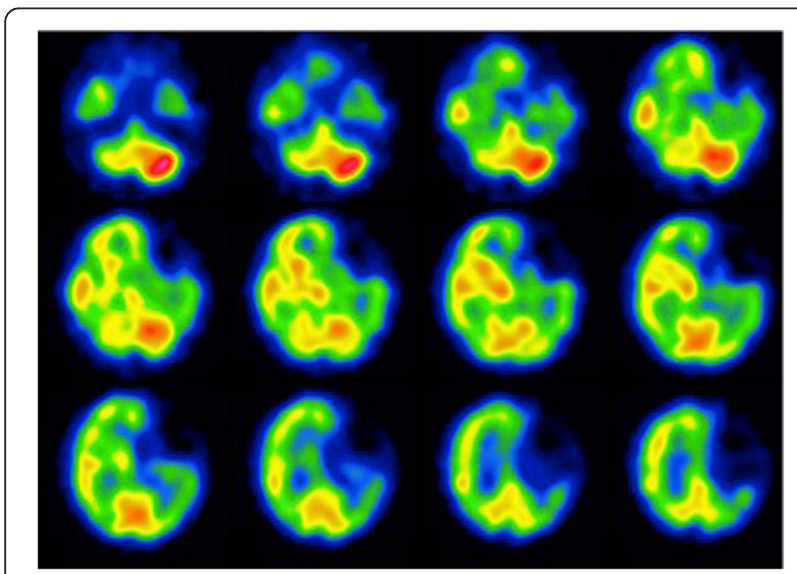

Fig. 1 Preoperative single-photon emission computed tomography scan. A preoperative single-photon emission computed tomography scan taken at the regional medical center revealed reduced cerebral blood flow in the left cerebral hemisphere (especially in the external left frontal cortex) right and left forehead (INVOS ${ }^{\mathrm{m}} 5100 \mathrm{C}$, Somanetics, USA) was initiated before the administration of general anesthesia. The $\mathrm{rSO}_{2}$ values for the left and right forehead were 72 and $81 \%$, respectively.

General anesthesia was induced by intravenous administration of $3 \mathrm{mg}$ of midazolam, $0.4 \mathrm{mg}$ of fentanyl, and $50 \mathrm{mg}$ of rocuronium. After tracheal intubation, a transesophageal echocardiography (TEE) probe was inserted. Then, a central venous catheter and right heart catheter were inserted via the right internal jugular vein, and central venous pressure, PAP, cardiac output, and mixed venous oxygen saturation were measured. The nasopharyngeal temperature and urinary bladder temperature were also measured.

Before $\mathrm{CPB}$, general anesthesia was maintained by inhalation of sevoflurane (1-1.5\% of end-tidal concentration). The patient's $\mathrm{PaCO}_{2}$ was maintained between 38 and $42 \mathrm{mmHg}$. Intra-aortic balloon pumping (IABP) was placed at the start of surgery and the augmented pressure was maintained. The mean arterial pressure was constantly above $70 \mathrm{mmHg}$. Before $\mathrm{CPB}, \mathrm{rSO}_{2}$ values were almost above $80 \%$ on both sides (Fig. 2).

During CPB, administration of sevoflurane via the oxygenator was also continued because of its cerebrovascular dilatation activity and potential preconditioning effect against focal cerebral ischemia. $\mathrm{PaCO}_{2}$ was maintained between 45 and $50 \mathrm{mmHg}$, and alpha-stat management of $\mathrm{pH}$ was performed. Hypothermia was induced; the temperature at the bottom of the nasopharyngeal temprature was $28{ }^{\circ} \mathrm{C}$. We used pulsatile perfusion assist to maintain cerebral circulation during $\mathrm{CPB}$ with IABP. A decrease in $\mathrm{rSO}_{2}$ was observed $162 \mathrm{~min}$ after the initiation of CPB. Our perfusionist increased the $\mathrm{CPB}$ pump flow from $2.2 \mathrm{~L} / \mathrm{min} / \mathrm{m}^{2}$ to $2.8 \mathrm{~L} / \mathrm{min} / \mathrm{m}^{2}$ in order to increase cerebral blood flow. Moreover, the concentration of sevoflurane was increased to $2 \%$. Yet, $\mathrm{rSO}_{2}$ desaturation (15\% reduction from baseline) was not improved. We decided to increase the depth-of-anesthesia with another dose of midazolam. After administration of $3 \mathrm{mg}$ of midazolam, the $\mathrm{rSO}_{2}$ values increased from 67 to $73 \%$ on the right side and from 71 to $74 \%$ on the left side. During $\mathrm{CPB}$, the lowest values (and variation) of $\mathrm{rSO}_{2}$ in the left and right forehead were $71 \%(-2 \%)$ and $67 \%(-17 \%)$, respectively. Mitral valve plasty was performed as planned.

At the weaning from the $\mathrm{CPB}$, the disappearance of MR was confirmed by TEE; the weaning was not difficult. Pulmonary hypertension also improved (PAP was $26 / 12 \mathrm{mmHg}$, while systemic arterial pressure was $105 / 56 \mathrm{mmHg}$ ). After CPB, inhalation of sevoflurane (1-1.5 \% of end-tidal concentration) was also continued. The $\mathrm{rSO}_{2}$ values were almost above $75 \%$ on both sides and not below the awake $\mathrm{rSO}_{2}$ values (Fig. 2). $\mathrm{CPB}$ and aortic cross-clamping lasted $352 \mathrm{~min}$ and $289 \mathrm{~min}$, respectively. On the completion of the surgery, the IABP 


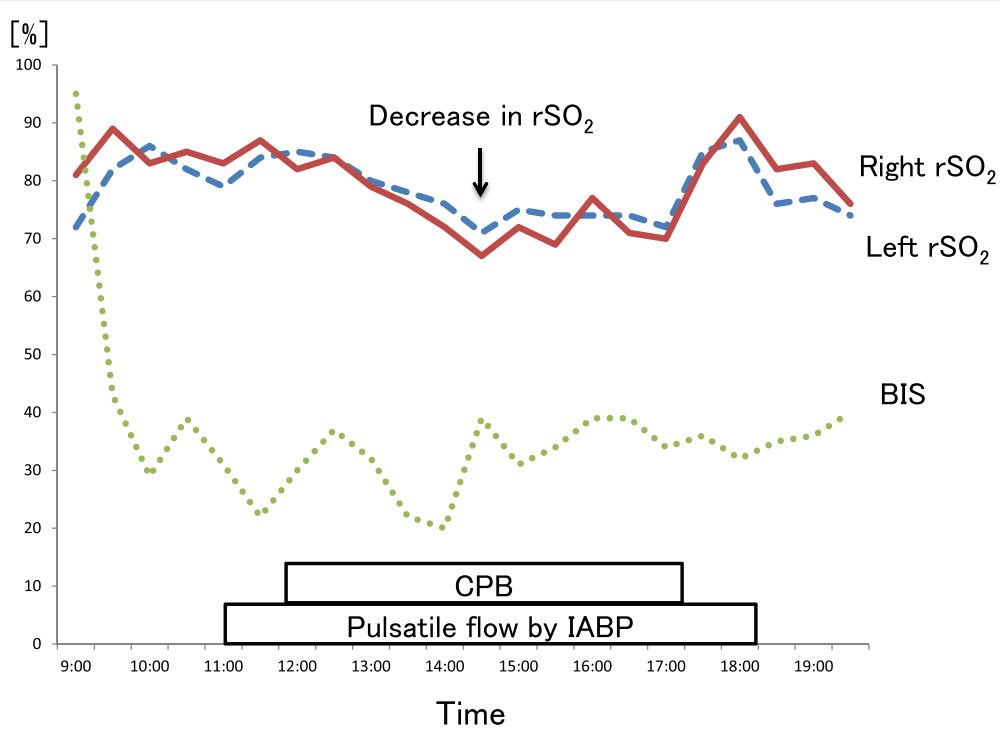

Fig. 2 Intraoperative regional cerebral oxygen saturation measured by $\mathrm{INVOS}^{\oplus}$ and bispectral index. $\mathrm{rSO}_{2}$, regional cerebral oxygen saturation; BIS, bispectral index; CPB, cardiopulmonary bypass; IABP, intra-aortic balloon pumping

was discontinued and sevoflurane administration was stopped. The patient was transferred to the intensive care unit with ventilator support under propofol sedation.

On the 1st postoperative day (POD), the patient was weaned from the ventilator, and the patient did not complain about any new neurological deficits. We monitored the $\mathrm{rSO}_{2}$ of his forehead until the 2nd POD and no significant decrease (-20\%) of the $\mathrm{rSO}_{2}$ values was confirmed. The postoperative course was uneventful. On the 15th POD, single-photon emission computed tomography revealed that the low cerebral blood flow lesions had not changed (Fig. 3), and the patient was discharged from our hospital on the 16th POD.

\section{Discussion}

We performed anesthesia for mitral valve plasty in a patient with cerebral infarction due to MMD using pulsatile perfusion of cardiopulmonary bypass with an assistance of IABP, without exacerbation of neurological complications.

Anesthetic management of a patient with MMD is rather challenging, and we have to keep several key-points in mind. Among them, maintaining normocapnea is most critical. Hypocapnia would induce brain ischemia and brain infarction, while hypercapnia would induce vasodilation and hyper-perfusion of the fragile vessels in the brain, which might cause brain hemorrhage. In patients with MMD, both vasoconstriction and vasodilation would not be preferable. That is why keeping normocapnea is quite important.
During $\mathrm{CPB}, \mathrm{PaCO}_{2}$ was maintained between 45 and $50 \mathrm{mmHg}$ with alpha-stat management of $\mathrm{pH}$. This patient developed MMD not by the onset of cerebral hemorrhage but by the onset of cerebral infarction. We regarded that we should avoid hypocapnia or normocapnea, which would induce cerebral infarction, rather than hypercapnia, which would induce cerebral hemorrhage. But, we cannot ignore that hypercapnia would induce vasodilation and hyper-perfusion of the fragile vessels in the MMD brain, which might cause brain hemorrhage.

Usually, extracorporeal circulation is maintained by non-pulsatile perfusion, and non-pulsatile perfusion can

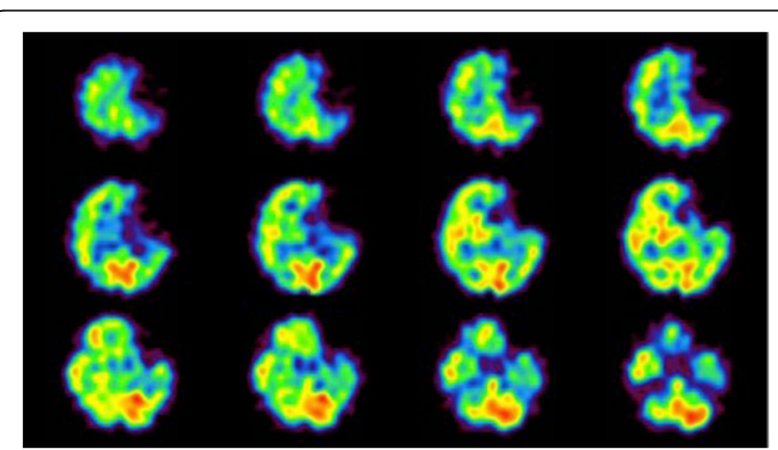

Fig. 3 Postoperative single-photon emission computed tomography (SPECT) scan. The postoperative SPECT scan obtained from our hospital revealed that low cerebral blood flow lesions in the left cerebral hemisphere had not changed compared to the preoperative SPECT scan 
induce ischemic injury, especially in organs under inadequate perfusion [5]. In patients with restricted cerebral arterial blood supply such as MMD, decreased cerebral perfusion pressure and non-pulsatile perfusion during $\mathrm{CPB}$ are risk factors for cerebral ischemia.

Kashima et al. [6] reported that high-pressure pulsatile perfusion assisted by IABP was effective for brain protection during coronary artery bypass grafting in a MMD patient. De Buysscher et al. [4] also reported that conversion from a non-pulsatile flow to a pulsatile flow resulted in a gradual increase in $\mathrm{rSO}_{2}$ values as opposed to a sudden decrease in $\mathrm{rSO}_{2}$ in an adult MMD patient undergoing CPB. In addition, Cheul-Hong et al. [3] reported up to $15 \%$ fluctuations in $\mathrm{rSO}_{2}$ values during cardiac surgery in an MMD patient who was later discharged from the hospital without any complications. In pediatric patients undergoing $\mathrm{CPB}$, pulsatile flow has advantages over nonpulsatile flow as measured by near-infrared spectroscopy and transcranial Doppler ultrasound, which may improve postoperative neurodevelopmental outcomes [7]. Owing to these previous reports, we selected the pulsatile flow method supported by IABP in our case and maintained the mean perfusion pressure above $70 \mathrm{mmHg}$.

The optimum anesthetic agent for the maintenance of anesthesia in MMD patients requiring cardiac surgery is a topic of much debate. Neither inhalational anesthetics (sevoflurane or isoflurane) nor intravenous anesthetic (propofol) presents strong clinical evidence for the efficacy of the outcome. Propofol suppresses cerebral metabolism and reduces cerebral blood flow [8]. In contrast, sevoflurane strongly dilates cerebral vessels and increases cerebral blood flow [9]. Moreover, several reports describe sevoflurane preconditioning against myocardial ischemia-reperfusion injury. Recently, it has been reported that sevoflurane has the potential preconditioning effect against cerebral ischemia [10], although obvious preconditioning effect of sevoflurane has not been proved clinically. In rats with transient cerebral ischemia, sevoflurane preconditioning protects mitochondria from cerebral ischemia-reperfusion injury and ameliorates long-term neurological deficits [11]. Thus, these properties may support sevoflurane use over propofol for intracranial steno-occlusive arterial disease, but choice of anesthetic in patients with MMD remains an open question.

A decrease in $\mathrm{rSO}_{2}$ was observed during the $\mathrm{CPB}$. Currently, $\mathrm{rSO}_{2}$ is considered as the average of arterial oxygen saturation $\left(\mathrm{SaO}_{2}\right)$ and internal jugular venous oxygen saturation $\left(\mathrm{SjO}_{2}\right)$ in the measured region. Several reasons should be considered for changes of $\mathrm{rSO}_{2}$. It is also known to be influenced by the skin blood flow [12]. Cutaneous vasoconstriction induced by vasoconstrictor, such as phenylephrine and norepinephrine, or hypothermia during $\mathrm{CPB}$ possibly affects changes of $\mathrm{rSO}_{2}$.
In this case, we increased the pump flow and concentration of sevoflurane to increase cerebral blood flow, but these interventions were not effective. Secondly, we deepened the anesthesia level with a supplemental intravenous anesthetic. After another administration of midazolam, an improvement in bilateral $\mathrm{rSO}_{2}$ values was observed. We didn't know why bilateral $\mathrm{rSO}_{2}$ was improved after administration of an intravenous anesthetic, but we cannot ignore the possibility that an intravenous anesthetic decreases the cerebral metabolic rate of oxygen consumption [7]. When regional cerebral oxygen desaturation continues during sevoflurane general anesthesia in patients with MMD, it might be efficacious to administer intravenous anesthetics concurrently. These management procedures limited the fluctuation of $\mathrm{rSO}_{2}$ values to within $17 \%$ in our patient, which was nearly equal to that in a previous study [3], and prevented the development of cerebral deficits.

\section{Conclusion}

Pulsatile flow supported by IABP during CPB had a possible beneficial effect for brain protection in patients with restricted cerebral arterial blood supply. Sevoflurane inhalation was continued during $\mathrm{CPB}$ with an expectation of its vasodilatory activity and its potential neuroprotective effect. We could maintain $\mathrm{rSO}_{2}$ at $80 \%$ of baseline value during the surgical procedure, and we could not detect other neurological deficits. But, the further discussion is warranted whether inhalational anesthetic or intravenous anesthetic might be superior for intracranial steno-occlusive arterial disease. Thus, our case provides further evidence for the efficacious use of this anesthetic in MMD patients requiring cardiac surgery.

\section{Abbreviations}

CPB, cardiopulmonary bypass; IABP, intra-aortic balloon pumping; MMD, moyamoya disease; $\mathrm{MR}$, mitral regurgitation; $\mathrm{PaCO}_{2}$, arterial carbon dioxide partial pressure; PAP, pulmonary arterial pressure; POD, postoperative day; $\mathrm{rSO}_{2}$, regional cerebral oxygen saturation; TEE, transesophageal echocardiography

\section{Acknowledgments}

We would like to thank Editage (www.editage.jp) for English language editing.

\section{Authors' contributions}

KS was primary anesthetist and drafted the manuscript. HT supervised anesthetic management. YE and MY helped to draft the manuscript. All authors read and approved the final manuscript.

\section{Authors' information}

KS is M.D., and Staff Anesthesiologists of Department of Anesthesiology, Tohoku University Hospital; HT is M.D., PhD, Lecturer of Department of Anesthesiology, Tohoku University Hospital; YE is M.D., PhD, and Associate Director Division of Surgical Center and Supply, Sterillization, Tohoku University Hospital; MY is M.D., PhD, and Professor of Anesthesiology and Perioperative Medicine, Tohoku University School of Medicine.

Competing interests

The authors declare that they have no competing interests. 


\section{Consent for publication}

Written informed consent was obtained from the patient for publication of this Case report and any accompanying images. A copy of the written consent is available for review by the Editor-in-Chief of this journal.

\section{Author details}

'Department of Anesthesiology, Tohoku University Hospital, 1-1 Seiryomachi, Aoba-ku, Sendai 980-8574, Japan. ${ }^{2}$ Division of Surgical Center and Supply, Sterillization, Tohoku University Hospital, 1-1 Seiryomachi, Aoba-ku, Sendai 980-8574, Japan. ${ }^{3}$ Anesthesiology and Perioperative Medicine, Tohoku University School of Medicine, 2-1 Seiryomachi, Aoba-ku, Sendai 980-8575, Japan.

Received: 17 March 2016 Accepted: 29 June 2016

Published online: 07 July 2016

\section{References}

1. Suzuki J, Takaku A. Cerebrovascular "moyamoya" disease. Disease showing abnormal net-like vessels in base of brain. Arch Neurol. 1969;20:288-99.

2. Wang N, Kuluz J, Barron N, et al. Cardiopulmonary bypass in a patient with moyamoya disease. Anesth Analg. 1997;84:1160-3.

3. Kim C-H, Yoon J-U, Lee H-J, et al. Hypothermic cardiopulmonary bypass for minimally invasive mitral valve plasty in adult moyamoya disease. J Anesth. 2012;26:259-61.

4. De Buysscher P, Moerman A, Bové T, et al. Value of cerebral oxygen saturation monitoring during cardiopulmonary bypass in an adult patient with moyamoya disease. J Cardiothorac Vasc Anesth. 2013;27:740-3.

5. Onorati $F$, Santarpino G, Presta $P$, et al. Pulsatile perfusion with intra-aortic balloon pumping ameliorates whole body response to cardiopulmonary bypass in the elderly. Crit Care Med. 2009;37:902-11.

6. Kashima I, Inoue Y, Takahashi R. The use of intra-aortic balloon pumping as cerebral protection in a patient with moyamoya disease undergoing coronary artery bypass grafting. Interact Cardio Vasc Thorac Surg. 2008;7:522-3.

7. Su XW, Guan Y, Barnes M, et al. Improved cerebral oxygen saturation and blood flow pulsatility with pulsatile perfusion during pediatric cardiopulmonary bypass. Pediatr Res. 2011;70:181-5.

8. Kaisti KK, Langsjo JW, Aslto S, et al. Effects of sevoflurane, propofol, and adjunct nitrous oxide on regional cerebral blood flow, oxygen consumption, and blood volume in humans. Anesthesiology. 2003;99:603-13.

9. Bundgaard $\mathrm{H}$, von Oettingen $\mathrm{G}$, Larsen $\mathrm{KM}$, et al. Effects of sevoflurane on intracranial pressure, cerebral blood flow and cerebral metabolism. A dose-response study in patients subjected to craniotomy for cerebral tumors. Acta Anaesthesiol Scand. 1998;42:621-7.

10. Codaccioni JL, Velly $L$, Moubarik C, et al. Sevoflurane preconditioning against focal cerebral ischemia: Inhibition of apoptosis in the face of transient improvement of neurological outcome. Anesthesiology. 2009;110:1271-8.

11. Ye R, Yang Q, Kong X, et al. Sevoflurane preconditioning improves mitochondrial function and long-term neurologic sequelae after transient cerebral ischemia: role of mitochondrial permeability transition. Crit Care Med. 2012;40:2685-93.

12. Sørensen $H$, Secher $\mathrm{NH}$, Siebenmann C, et al. Cutaneous vasoconstriction affects near-infrared spectroscopy determined cerebral oxygen saturation during administration of norepinephrine. Anesthesiology. 2012;117:263-70.

\section{Submit your manuscript to a SpringerOpen ${ }^{\mathcal{O}}$ journal and benefit from:}

- Convenient online submission

- Rigorous peer review

- Immediate publication on acceptance

- Open access: articles freely available online

- High visibility within the field

- Retaining the copyright to your article 